\author{
Original Article $\quad$ www.pjkd.com.pk
}

\title{
One Year Reinfection Rate of Hepatitis C Among Patients on Maintenance Hemodialysis After Successful Antiviral Treatment
}

\author{
Sanaa Khurshid ${ }^{1}$, Sidra Shafiq Cheema ${ }^{2}$, Shafiq Cheema ${ }^{1}$, \\ ${ }^{1}$ Department of Nephrology - Jinnah Hospital/ Allama Iqbal Medical College, Lahore, \\ Pakistan, \\ 2Department of Pathology-Combined Military Hospital, Lahore Medical College, Lahore, \\ Pakistan.
}

\begin{abstract}
Background: The use of dedicated dialysis machines for $\mathrm{HCV}$-infected patients is not recommended but hemodialysis units should ensure implementation of, and adherence to, strict infection control procedures designed to prevent transmission of blood borne pathogens. Direct acting antiviral therapies (DAA) are an important tool for hepatitis $\mathrm{C}$ virus elimination. However, reinfection among high risk population especially hemodialysis patients may hamper the elimination targets. The most important factor implicated in HCV transmission in dialysis unit is cross-contamination from supplies and surfaces as a result of failure to follow infection control procedures. Therefore, we estimated $\mathrm{HCV}$ reinfection rates among treated individuals on maintenance hemodialysis (MHD) in our dialysis center at Jinnah hospital.

Aim: The primary objective of this study is to determine the rates of HCV reinfection after successful treatment, among patients on MHD. Methods: We analyzed data from Jinnah hospital dialysis center cohort on maintenance hemodialysis who previously took direct acting antiviral therapy (DAA) for treatment of hepatitis $\mathrm{C}$ in a prospective randomized interventional trial which included 36 patients. In this trial, group 1(18 patients) received $400 \mathrm{mg}$ daily sofosbuvir/ $60 \mathrm{mg}$ daily daclatasvir; while group 2 (18 patients) received thrice a week $400 \mathrm{mg}$ sofosbuvir/ daily $60 \mathrm{mg}$ daclatasvir for 12 weeks. These patients were followed for one year after sustained virologic response (SVR) was achieved. During this time 'universal precautions' to prevent cross-contamination of HCV were implemented. HCV RNA PCR was measured at one year again. Reinfection was defined as a positive HCV RNA measurement at one year after achievement of SVR. Genotype was also assessed for patients who had positive HCV RNA by PCR. Crude reinfection rates per 100 person-years were calculated. As a secondary objective, one year mortality was also calculated in this treated population.

Results: Of 32 patients who were treated with DAAs, SVR after one year of completion of treatment was $100 \%$ in group 1 and $85.8 \%$ in group 2. Overall SVR was $86.3 \%$. None of the patients in group 1 had reinfection one year after achieving SVR while 2 out of 14 patients in group 2 were found to have reinfection with same genotype as previous. Overall reinfection rate was 2/32 (6.25\%). Crude reinfection rate per 100 person-years was 6.25. One year mortality was 16\% and $44 \%$ in group 1 and 2 respectively.

Conclusion: Reinfection rate is low among successfully treated HCV patients on maintenance hemodialysis when strict implementation and adherence to 'universal precautions' is practiced.

Key words: Hepatitis $C$ virus, seroconversion, direct acting antiviral therapy, hemodialysis, universal precautions, sustained virologic response.

\section{Corresponding Author}

Professor Dr. Shafiq Cheema, MBBS, DABN, DAIM, FACP, FASN (USA)

Department of Nephrology,

Jinnah Hospital/ Allama Iqbal Medical College,

Lahore, Pakistan.

Email: shafiqcheema@yahoo.com

Received: April 27, 2020. Accepted July 27, 2020.

PJKD 2020;4(3):290-293

\section{Introduction:}

Hepatitis $\mathrm{C}$ virus $(\mathrm{HCV})$ infection is a global health and economic problem and hemodialysis has always been a risk factor for acquiring $\mathrm{HCV}$ infection, as shown by numerous outbreaks and $\mathrm{HCV}$ cross-infections that have occurred in hemodialysis units. ${ }^{1.3}$ Earlier studies conducted have shown an HCV prevalence in hemodialysis patients that ranged from 2.6 to $60 \%$ (10-fold higher than the general population), with higher prevalence correlating with longer duration of hemodialysis. ${ }^{46}$

The risk of HCV transmission in hemodialysis units has declined over years due to improved testing and infection control practices. ${ }^{7.8}$ Several studies have shown that patients on chronic hemodialysis have an increased overall mortality risk if they have chronic hepatitis $\mathrm{C}$ infection. ${ }^{9,10}$ There are also some data showing that chronic hepatitis $\mathrm{C}$ may be a risk factor for developing renal cell carcinoma and increases the risk of serious infections in renal transplantation recipients. ${ }^{11,2}$ Pegylated- interferon alone or in combination with ribavirin (RBV) have been the mainstay of treatment for HCV infection in hemodialysis patients but is associated with longer treatment duration, poor virologic response with low efficacy, lesser tolerability, high frequency of adverse effects, and requires close
\end{abstract}




\section{Reinfection in Treated HCV Hemodialysis Patients}

supportive care. ${ }^{13}$ The availability of direct-acting antiviral agents (DAAs) has sparked major enthusiasm for treating persons with HCV who have chronic renal impairment, especially since many of these individuals have not been eligible for treatment given the toxicities associated with interferon and ribavirin-based therapy. ${ }^{14}$ Since HCV infection negatively impacts dialysis patients in terms of morbidity and mortality compared with non-HCV dialysis patients, effective treatment is especially essential. New generation DAAs like NS5B inhibitor sofosbuvir (SOF) combined with NS5A inhibitor daclatasvir (DCV), with or without ribavirin (RBV) have already been shown to be highly effective and tolerable in treating HCV infection in patients on maintenance hemodialysis. After establishing the fact that direct-acting antiviral agents are an important tool for hepatitis $C$ virus elimination in hemodialysis population, the next step is to measure the reinfection rates in this high risk population as reinfection may hamper the elimination target. In HD facilities, the most common lapses of healthcare quality are contamination of dialysis systems, inadequate disinfection and cleaning of environmental surfaces, improper contact of health care staff with equipment and patients, and mishandling of parenteral medications. ${ }^{15,16}$ Therefore, this study is designed to determine reinfection rates after successful treatment with DAAs in hemodialysis population provided strict infection control practices are applied.

\section{Patients and Methods:}

Trial Design and Participants

We analyzed data from the same cohort who previously took direct DAA for treatment of hepatitis $\mathrm{C}$ in a prospective randomized interventional trial including 36 patients. ${ }^{17}$ In this trial, group $1(18$ patients) received $400 \mathrm{mg}$ daily sofosbuvir/ $60 \mathrm{mg}$ daily daclatasvir; while group 2 (18 patients) received thrice a week $400 \mathrm{mg}$ sofosbuvir/ daily $60 \mathrm{mg}$ daclatasvir for 12 weeks and patients with compensated cirrhosis were given treatment for 24 weeks (shown in figure).

Outcomes and Follow Up

These 32 treated patients were followed for one year after sustained virologic response (SVR) was achieved. During this time 'universal precautions' to prevent cross-contamination of HCV were implemented. HCV RNA PCR was measured at one year again. Reinfection is generally defined as a case in which an initial infection is completely resolved prior to a subsequent infection. This can be either a reinfection with a different genotype/subtype compared to the initial infection, or with the same subtype but a different strain. Reinfection in this follow up was defined as a positive HCV RNA measurement at one year after achievement of SVR. Genotype was also assessed for patients who had positive HCV RNA by PCR to assess whether it is a reinfection or cross over infection. Crude reinfection rates per 100 person-years were calculated. As a secondary objective we also calculated one-year mortality in this treated population.

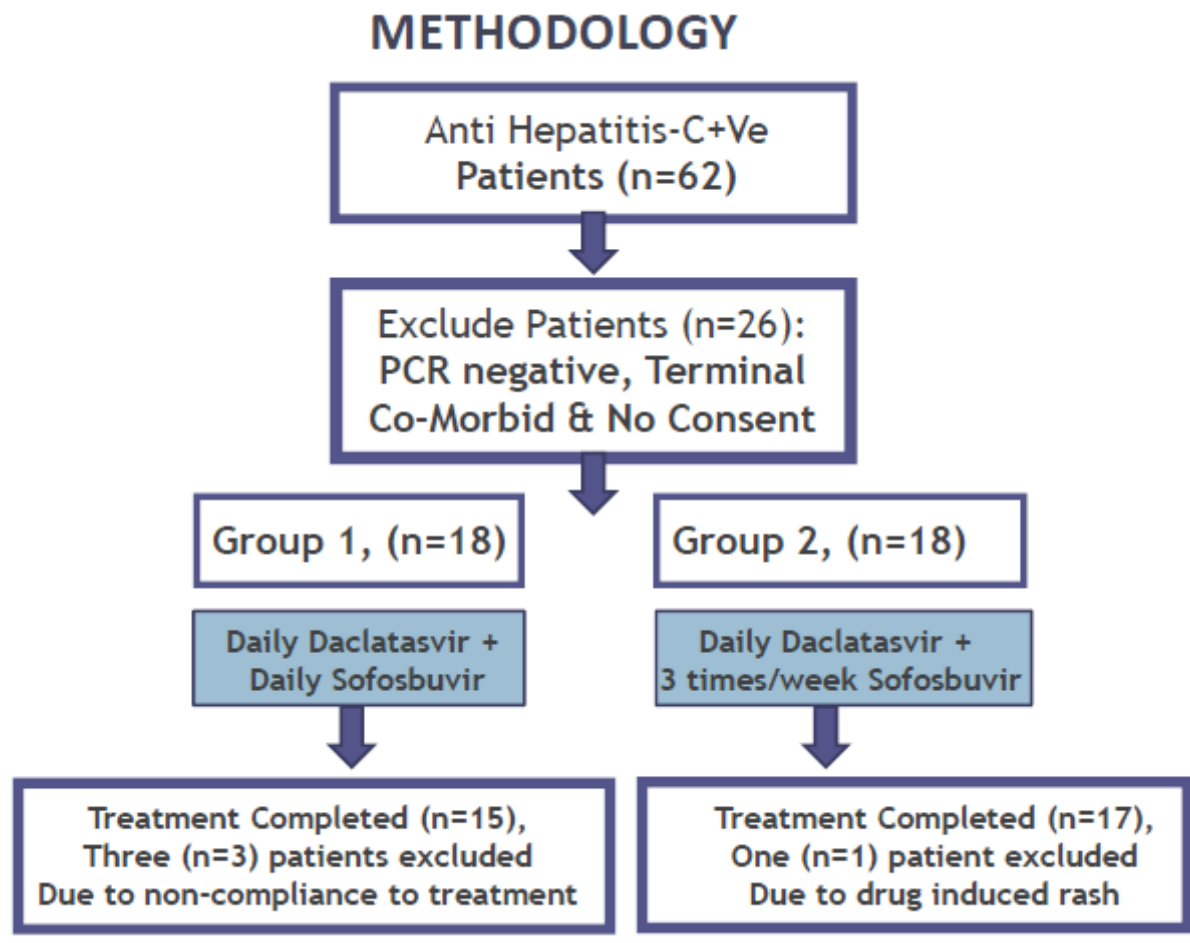

Results:

Of these 32 treated patients, none of the patients in group 1 had reinfection one year after achieving SVR while 2 out of 14 patients in group 2 were found to have reinfection with same genotype as previous one. SVR after one year of completion of treatment was $100 \%$ in group 1 (daily sofosbuvir and daclatasvir) and $85.8 \%$ in group 2 (daily daclatasvir and thrice weekly sofosbuvir). Overall SVR was $86.3 \%$. Overall reinfection rate was 2/32 (6.25\%). Crude reinfection rate was 6.25 cases per 100 person years. One-year mortality was $16 \%$ and $44 \%$ in group 1 and 2 respectively with overall mortality of $30 \%$. 


\section{Reinfection in Treated HCV Hemodialysis Patients}

\section{Discussion:}

$\mathrm{HCV}$ is endemic in Pakistan and 6.8\% population of Pakistan may be infected with HCV which is almost $40 \%$ increase in HCV seroprevalence among the general population in recent years. ${ }^{18,19}$ The high sustained virologic response rate with the newer medications has been found in populations typically considered difficult-to-treat. The newer DAAs which can be safely used in patients with advanced CKD are not yet available in Pakistan and options are further limited as about $80-90 \%$ of the patients are infected with genotype 3 . $^{20}$ SOF based regimens are backbone of current regimen to treat HCV and once-daily oral daclatasvir plus sofosbuvir was associated with high rates of sustained virologic response among hemodialysis patients infected with HCV genotype 1, 2, or 3. ${ }^{17}$ This background study is by far the largest study from Pakistan showing that SOF based therapy can be safely and effectively used to treat HCV in patients undergoing MHD and to found the re-infection in this high risk population is also the first follow up study.

$\mathrm{HCV}$ reinfection is a particular concern in patients, who continue to engage in high risk behaviors such as injection drug use, HIV/HCV co infection. ${ }^{21}$ Similarly, hemodialysis patients are also considered as high-risk population. Since intravenous drug use remains the leading cause of $\mathrm{HCV}$ infection and reinfection, several studies have addressed HCV reinfection following treatment-induced clearance among people who inject drugs. ${ }^{22}$ In Vancouver, the incidence of first HCV infection was 7.3 cases per 100 person years and the risk of reinfection after HCV treatment was 3.2 cases per 100 person years. ${ }^{23}$ In Amsterdam, the local incidence of first HCV infection of 0.35 cases per 100 person years, significantly lower than in Vancouver and the risk of reinfection after HCV treatment was 0.76 cases per 100 person years. ${ }^{24}$ In another Australian study, incidence of potential HCV reinfection among participants with HCV clearance with 42 per 100 person years (95\% CI, 25 - 61/100 PY) was comparable to the incidence of initial HCV infection among HCV negative participants. ${ }^{25}$

A study from Spain followed 118 prisoners (81\% injection drug users) treated for HCV who had achieved a sustained virologic response between 2003 and 2009 and found that the incidence of reinfection was high, especially among those who reported ongoing injection drug use. ${ }^{26}$ At follow-up, HCV reinfection was identified in nine former injection drug users; seven with an HCV genotype switch - for an overall reinfection rate of 5.27 cases per 100 person years. The incidence of reinfection was significantly higher among people who were actively using drugs (hazard ratio $\mathrm{HR}=12.47 ; 95 \% \mathrm{CI}: 2.90-53.71$ ), people who were HIV co-infected $(\mathrm{HR}=9.95 ; 95 \% \mathrm{CI}: 1.73-$ 57.34) and those engaging in more than one risk behavior after treatment ( $\mathrm{HR}=7.47$; 95\% CI: 1.19-46.89).

The metanalysis of these studies concluded that in HCV mono-infected patients with no recognized risk factors, the pooled estimate of reinfection rate and summary 5-year reinfection risk were zero. ${ }^{21}$ In HCV mono-infected patients engaging in activities that are higher risk, the pooled reinfection rate was $19.06 / 1000$ person years $(95 \% \mathrm{CI}, 11.42-28.16)$ leading to a summary five-year risk of $10.67 \%(95 \% \mathrm{CI}$, $6.38 \%$ - 15.66\%).In HIV/HCV co-infected patients, the pooled reinfection rate was 32.02/1000 person years (95\% CI, $0.00-123.49)$ leading to a summary five-year risk of $15.02 \%(95 \% \mathrm{CI}, 0.00 \%-48.26 \%)$. The review concluded that most patients continue to have a sustained virologic response (i.e. no reinfection) five years post-treatment. The greater reinfection risk among people engaging in higher risk activities and among people with HIV-HCV co-infection highlight the need for prevention campaigns specifically targeted at these groups. Similarly, in our study the pooled reinfection rate in HCV mono-infected patients on hemodialysis was 2/32 (6.25\%) comparable with other high-risk population and crude reinfection rate per person year was 0.0624 . Therefore, the focus should be on prevention strategies.

Hemodialysis units have responsibility to ensure implementation of, and adherence to, strict infection-control procedures designed to prevent nosocomial transmission of blood-borne pathogens, including HCV (strong evidence) between patients in their care, either directly or via contaminated equipment or surfaces. Recommendations of National kidney foundation include dialysis unit design should facilitate implementation of infection control strategies, time between shifts should be sufficient to enable effective machine and surface decontamination, ease of disinfection should be a consideration when selecting new equipment, ensure that infection-control staff training and vigilance is maintained during changes to staff-to-patient ratios or employment of new staff, carry out regular risk assessments and develop procedures to reduce or remove hazards. Isolating HCV-infected patients as an alternative to strict infection-control procedures for preventing transmission of blood-borne pathogens (weak evidence), using dedicated dialysis machines for HCV-infected patients (moderate evidence) is not recommended by National kidney foundation.

\section{Conclusion}

Reinfection rate is low among successfully treated HCV patients on maintenance hemodialysis when strict implementation and adherence to 'universal precautions' is practiced. HCV RNA negative patients can also be dialyzed in the same unit with other HCV RNA positive patients and dedicated HCV negative machines is not needed. Mortality remains high in this cohort consistent with several meta-analyses concluding that $\mathrm{HCV}$ is an independent and significant risk factor for death in ESRD patients on dialysis.

\section{Conflict of Interest: None declared}

\section{Referrences}

1. Wreghitt TG. Blood-borne virus infections in dialysis units--a review. Rev Med Virol. 1999;9:101-9.

2. Thomson PC, Williams C, Aitken C, Ball J, Wysocka N, Brown R et al. A case of hepatitis C virus transmission acquired through sharing a haemodialysis machine. NDT Plus. 2011;4:32-5

3. Muleta D, Kainer MA, Moore-Moravian L, Andrew, Wiese A, Ward J, McMaster S et al. Notes from the Field: Hepatitis C Outbreak in a Dialysis Clinic--Tennessee, 2014. MMWR Morb Mortal Wkly Rep. 2016;64:1386-7.

4. Finelli L, Miller JT, Tokars JI, Alter MJ, Arduino MJ. National surveillance of dialysis-associated diseases in the United States, 2002. Semin Dial. 2005;18:52-61. 


\section{Reinfection in Treated HCV Hemodialysis Patients}

5. Fissell RB, Bragg-Gresham JL, Woods JD, Jadoul M, Gillespie B, Hedderwick SA et al. Patterns of hepatitis C prevalence and seroconversion in hemodialysis units from three continents: the DOPPS. Kidney Int. 2004;65:2335.

6. Carbone M, Mutimer D, Neuberger J. Hepatitis C virus and nonliver solid organ transplantation. Transplantation. 2013;95:779-86.

7. Patel PR, Thompson ND, Kallen AJ,Arduino MJ. Epidemiology, surveillance, and prevention of hepatitis C virus infections in hemodialysis patients. Am J Kidney Dis. 2010;56

8. Agarwal SK, Dash SC, Irshad M, Gupta S, Bohmik D, Tieari SC et al. Impact of hepatitis C virus infection on renal transplant outcome in India:a single centre study. J Assoc Physicians India. 2000;48:1155-1159.

9. $\quad$ Carbone M, Cockwell P, Neuberger J. Hepatitis C and kidney transplantation. Int J Nephrol. 2011;593291:1-17.

10. Fabrizi F, Takkouche B, Lunghi G, Dixit V, Messa P, Martin P. The impact of hepatitis C virus infection on survival in dialysis patients: metaanalysis of observational studies. J Viral Hepat. 2007;14:697-703.

11. Gordon SC, Moonka D, Brown KA, Charat Thongprayoon, Natanong Thamcharoen, Kunlatida Maneenil et al. Risk for renal cell carcinoma in chronic hepatitis C infection. Cancer Epidemiol Biomarkers Prev. 2010;19:1066-73

12. Gonzalez HC, Lamerato L, Rogers CG, Gordon SC. Chronic Hepatitis C Infection as a Risk Factor for Renal Cell Carcinoma. Dig Dis Sci 2015;60(6):1820-4

13. KDIGO Work Group. Guideline 4: Management of HCV infected patients before and after kidney transplantation. Kidney Int. 2008;73:553-68.

14. Maruyama A, Partovi N, Yoshida EM, Erb SR, Azalgara VM, Hussaini T. A review of direct-acting antivirals for the treatment of hepatitis C in patients with advanced chronic kidney disease. Nephrol Dial Transplant. 2017;32:35-41.

15. Recommendations for preventing transmission of infections among chronic hemodialysis patients. MMWR Recomm Rep. 2001;50:1-43.

16. Bianco A, Bova F, Nobile CG, Pileggi C, Pavia M. Healthcare workers and prevention of hepatitis C virus transmission: exploring knowledge, attitudes and evidence-based practices in hemodialysis units in Italy. BMC Infect Dis. 2013;13:76.

17. Cheema SR, Rehman MS, Hussain G, Cheema SS, Gilani N. BMC Nephrology, efficacy and tolerability of sofosbuvir and daclatasvir for treatment of hepatitis C genotype $1 \& 3$ in patients undergoing hemodialysis. 2019;20:438.

18. Zampieron A, Jayasekera H, Elseviers M, Lindley E, DeVos JY, Visser R, Harrington M. European study on epidemiology and management of hepatitis $\mathrm{C}$ virus (HCV) infection in the haemodialysis population. Part 3: prevalence and incidence. EDTNA ERCA J. 2006;32:42-44.

19. Umer M, Iqbal M. Hepatitis C virus prevalence and genotype distribution in Pakistan: Comprehensive review of recent data. World J Gastroenterol. 2016;22:1684-1700.

20. Elbaz T, El-Kassas M, Esmat G. New era for management of chronic hepatitis C virus using direct antiviral agents: A review. Journal of advanced research 2015;6(3):301-10.

21. Simmons B, Saleem J, Hill A, Riley RD, Cooke GS. Risk of late relapse or reinfection with hepatitis C virus after achieving a sustained virological response: A systematic review and metaanalysis. Clinican Infectious Diseases 2016;62(6):683-94.

22. Grady BP, Schinkel J, Thomas XV, Dalgard O. Hepatitis C virus reinfection following treatment among people who use drugs. Clinical Infectious Diseases 2013;57(Suppl 2):S105-S110.

23. Grebely J, Knight E, Ngai T, Genoway KA, Raffa JD, Storms M, et al. Reinfection with hepatitis C virus following sustained virological response in injection drug users. Journal of Gastrointestinal and Hepatology 2010;25(7):1281-4.

24. Grady BP, Vanhommerig JW, Schinkel J, Weegink CJ, Bruisten SM, Lindenburg CE, et al. Low incidence of reinfection with the hepatitis C virus following treatment in active drug users in Amsterdam. European Journal of Gastroenterology \& Hepatology 2012;24(11):1302-7.

25. Micallef JM, Macdonald V, Jauncey M, Amin J, Rawlinson W, van BI, et al. High incidence of hepatitis C virus reinfection within a cohort of injecting drug users. Journal of Viral Hepatitis 2007;14(6):413-8.

26. Marco A, Esteban JI, Sole C, da SA, Ortiz J, Roget M, et al. Hepatitis C virus reinfection among prisoners with sustained virological response after treatment for chronic hepatitis C. Journal of Hepatology 2013;59(1):45-51. 